\title{
Physically-Informed Correction of Instability (Drift)
}

\author{
Shahriar Jamasb, Member, IACSIT
}

\begin{abstract}
A Physical model for the long-term deterministic behavior associated with instability (or drift) in $\mathrm{pH}$-sensitive ion-selective field effect transistors (ISFET's) has been employed to distinguish between drift and the inherent low-frequency $(1 / f)$ noise, both of which corrupt the sensor output signal. Based on this model, a physically-informed method for sensor signal enhancement in the time-domain has been proposed, which relies on proper windowing of the sensor output. The random-like evolution in time associated with a weak power-law relaxation towards equilibrium is the key aspect of the model for drift that is utilized in the proposed corrective scheme. Drift may be interpreted as a weakly chaotic behavior, exhibiting anomalous dynamics characterized by ageing, which reflects a weak (power-law) relaxation towards equilibrium.
\end{abstract}

Index Terms-1/f Noise, drift, instability, ISFET, physically-informed signal processing, power-law, weak chaos

\section{INTRODUCTION}

In biomedical applications involving continuous monitoring sensor accuracy is of paramount importance. Instability (drift) and inherent noise impose fundamental limitations on sensor accuracy. Drift is often ascribed to aging of sensor materials, and manifests as a relatively slow, long-term, temporal change in the sensor output in absence of variations in the measurand (i.e. stimulus). In continuous monitoring applications drift represents a more serious challenge necessitating frequent recalibrations. Fundamental sources of noise such as thermal noise and low frequency (1/f) noise may also significantly limit sensor accuracy if small variations in the measurand are to be detected. White noise, however, can be easily distinguished from frequency dependent disturbances.

Sensor instability or drift typically manifests complex dynamics characterized by a random-looking evolution in time following an initial relatively fast transient. While the physical mechanisms underlying inherent noise are relatively well understood, novel properties such as ageing of sensor materials commonly ascribed to drift exhibit complex dynamics. A case in point is represented by the $\mathrm{pH}$-sensitive ion selective field effect transistor (ISFET), a solid state device which combines the $\mathrm{pH}$-sensing properties of an insulator such as silicon nitride $\left(\mathrm{Si}_{3} \mathrm{~N}_{4}\right)$ or aluminum oxide $\left(\mathrm{Al}_{2} \mathrm{O}_{3}\right)$ with the field sensing characteristics of a FET. ISFET is subject to inaccuracies originating from both drift and low frequency (1/f) noise. The $1 / \mathrm{f}$ noise mechanism in metal-oxide-semiconductor FET (MOSFET) has been studied extensively and is rather well elucidated [1]-[4]. In

Manuscript received October 13, 2012; revised November 12, 2012.

Shahriar Jamasb is with the Biomedical Engineering Department, Hamedan University of Technology, Hamedan, Hamedan 65169-13418 Iran (e-mail: jamasbs@profs.hut.ac.ir). contrast, very little effort has been made to develop physical models for drift. A physical model for ISFET drift, however, has been presented [5], [6], [7] and the distinction between drift and 1/f noise behavior in ISFET's has also been elucidated [8]. In this work the proposed drift model, which quantitatively accounts for the nonlinear dynamical phenomenon of ISFET drift, is briefly introduced in order to demonstrate its utility in a physically-informed method for sensor signal enhancement. Revisiting the physical model for drift in order to explore the possibility of formulation of the model using stochastic differential equations can be useful for distinguishing the nonlinear dynamical behavior of the device from random processes. However, here, only the distinction between the long-term deterministic drift behavior and the low frequency (1/f) noise characteristics in ISFET's is demonstrated to support the argument that the physical mechanisms responsible for $1 / \mathrm{f}$ noise and drift are entirely different. Such distinction is also shown to be relevant to the proposed method for extraction of the deterministic measuring signal associated with sensor transfer function. Finally, implications of the nonlinear dynamical drift behavior, which may be of relevance to physically-informed processing of sensor signals, are explored

\section{THE PHYSICAL MODEL FOR ISFET DRIFT}

The instability or drift in an $\mathrm{N}$-channel ISFET is characterized by a relatively fast transient increase, followed by a long-term temporal increase in the threshold voltage of the device, which leads to a corresponding decrease in the drain current. The origin of the nonlinear dynamical behavior associated with ISFET drift can be explained based on anomalous diffusion occurring during a phase transition phenomenon. In particular, chemical modification of the surface of the pH-sensing insulator (e.g. $\mathrm{Al}_{2} \mathrm{O}_{3}$ ), which is exposed to the electrolyte solution, leads to formation of a hydrated layer whose rate of growth is modeled by implementing a hopping and/or trap-limited transport mechanism, known as dispersive diffusion [5,6,7]. Dispersive diffusion is characterized by a power-law time decay of mobility or diffusivity of the form $t^{\beta-1}$, where $\beta$ is the dispersion parameter satisfying $0<\beta<1$ [9]. This unique time-dependence has been explained based on the concept of a continuous-time random walk whose origin is either a) hopping motion through localized states giving rise to hopping transport or b) multiple trapping from a band of localized states leading to multiple-trap transport in disordered materials (e.g. $\mathrm{Si}_{3} \mathrm{~N}_{4}$ or $\mathrm{Al}_{2} \mathrm{O}_{3}$ ) [10]. Specifically, the time dependence of the random walk is determined by a broad distribution of event times, which are identified as hopping times and trap release times in hopping transport and 
multiple-trap transport mechanisms respectively. The distribution of these event times may be regarded as the origin of the fluctuations in the ISFET drain current associated with drift following the initial relatively fast transient. This random-like temporal evolution is clearly evident on a time scale of a few seconds. Dispersive diffusion of hydrating species within the amorphous structure of the $\mathrm{Si}_{3} \mathrm{~N}_{4}$ leads to a decay in the density of sites/traps occupied by the diffusing species [6]. For small departures from equilibrium based on a linear approximation, which takes the power-law time dependence of dispersive diffusion into account, the decay in the density of sites/trap is described by a stretched-exponential relaxation of the form $\exp \left[-(t / \tau)^{\beta}\right]$, where the time constant $\tau$ is the time required for structural relaxation, obeying an Arrhenius relationship [9]. A closed-form expression for the growth of the hydration layer at the interface between the electrolyte solution and the insulator, therefore, can be derived [6]:

$$
x_{H L}(t)=x_{H L}(\infty)\left\{1-\exp \left[-(t / \tau)^{\beta}\right]\right\}
$$

where $x_{H L}$ represents the thickness of the hydrated layer. Furthermore, it has been shown [6] that the time dependence of ISFET drift is identical to that associated with the growth of the hydration layer. As shown in Fig.1, the proposed model accurately describes the long-term, deterministic drift behavior in an $\mathrm{Al}_{2} \mathrm{O}_{3}$-gate ISFET despite the fluctuations in the drain current, $I_{D}$, on short time scales.

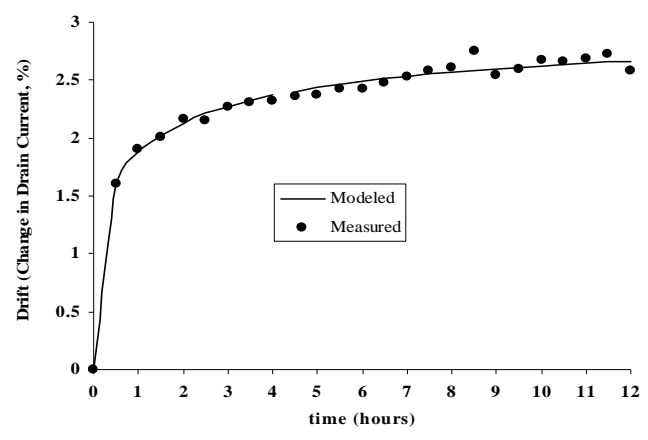

Fig. 1 Modeled versus Measured ISFET Drift, $\mathrm{pH}=7$

TABLE I: DEPENDENCE OF DRIFT ON INITIAL CONDITIONS

\begin{tabular}{|c|c|c|c|}
\hline \multirow{2}{*}{$\begin{array}{c}\text { pH of } \\
\text { Buffer }\end{array}$} & \multicolumn{3}{|c|}{ Drift Model Parameters } \\
\cline { 2 - 4 } & $x_{H L}(\infty)$ (angstrom & $\tau$ (hours) & $\beta$ \\
\hline 7 & 105 & 53.45 & 0.613 \\
\hline 10 & 124 & 66.46 & 0.358 \\
\hline
\end{tabular}

The modeled-versus-measured fit depicted in Fig.1 is characterized by a coefficient of correlation of 0.99 with extracted parameters $I_{D}(\infty)=673.90 \mu \mathrm{A}$ and $\beta=0.355$. In addition, it is important to note that ISFET drift characteristics are highly sensitive to initial conditions. As indicated in Table I, the extracted model parameters for a $\mathrm{Si}_{3} \mathrm{~N}_{4}$-gate ISFET at two different $\mathrm{pH}$ values exhibit a relatively wide variation. Etching the insulator surface following exposure of the $\mathrm{pH}$-sensitive insulator to the solution and the consequent removal of the hydration layer, also leads to a significant variation in the drift characteristics and the corresponding model parameters. This indicates that the initial surface condition is an important factor in determining the drift dynamics.

\section{PHYSICALLY INFORMED CORRECTION OF ISFET DRIFT}

The long-term deterministic model for ISFET drift indicates that following an initial, relatively fast transient, the drift is characterized by a significantly slower and irregular temporal evolution, which does not quite reach equilibrium as, $t \rightarrow \infty$. Therefore, following the initial, fast transient resulting from drift, sensor signal enhancement may be possible by cutting the measuring signal (i.e. the device output) into sufficiently short-duration segments over which a relatively fast deterministic response associated with sensor transfer function is detectable. In fact, this idea has been successfully employed for correction of drift in $\mathrm{pH}$-sensitive ISFET's [11], [12], [13]. Essentially, this corrective scheme is equivalent to deriving the sensor response by integrating the differential of the output signal arising solely from changes in the measurand. As shown in Fig. 2, the proposed method allows accurate continuous monitoring of $\mathrm{pH}$ under conditions approximating acute metabolic acidosis.

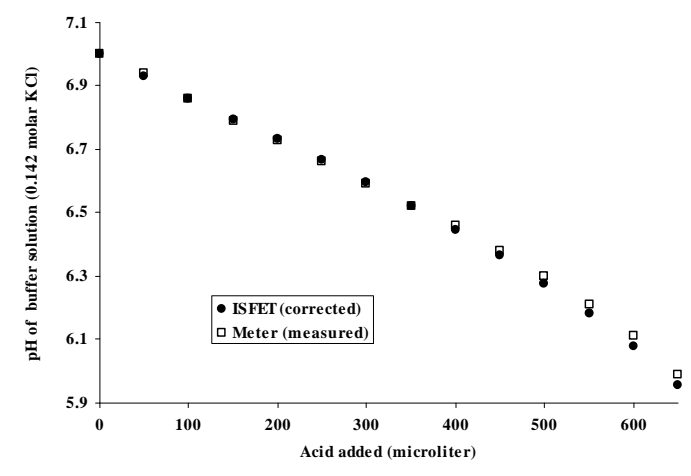

Fig. 2. Measured versus corrected $\mathrm{pH}$

In Fig. 2, the decrease in $\mathrm{pH}$ simulating a worsening metabolic acidosis condition was induced by adding 50 microliters of 2-normal hydrochloric acid to the solution at 5-minute intervals, and the device output was monitored over segments of 3-second duration [12], [13]. During this experiment, the $\mathrm{pH}$ was also monitored using a Piccolo Plus $\mathrm{pH}$ meter with an accuracy of $\pm 0.01 \mathrm{pH}$ unit. The requirement for the validity of this method, however, is that the drift rate is significantly lower than the product of the device sensitivity and the rate of change of the measurand. For a $\mathrm{Si}_{3} \mathrm{~N}_{4}$-gate $\mathrm{pH}$-sensitive ISFET the typical long-term drift rate (i.e. following the initial fast transient) and the device sensitivity values are 0.0975 microampere per hour and 4.23 microampere per $\mathrm{pH}$ unit at neutral $\mathrm{pH}$. This implies that the above requirement for validity of the proposed method is readily satisfied for $\mathrm{pH}$ variations occurring at rates higher than $0.20 \mathrm{pH}$ unit per hour [11].

The choice of the appropriate signal segment duration (i.e. time interval), over which variations in the sensor output signal arise solely from drift, can be justified by distinguishing between drift and low frequency noise. Such a distinction can be made using the proposed physical model 
for drift. In particular, given the accuracy of the analytical formulation of this model (coefficients of correlation better than 0.999), the output noise spectrum can be readily extracted from the measured output characteristics of the ISFET at a fixed $\mathrm{pH}$. For an ISFET operating in the feedback mode (i.e. at a constant drain current), the output noise can be written as

$$
s_{n}(t)=s_{0}(t)-s_{d r i f t}(t)
$$

where $s_{n}(t), s_{0}(t), s_{\text {drift }}(t)$ are the signals associated with the noise, the device output at fixed $\mathrm{pH}$, and the modeled drift characteristics respectively. The ISFET input-referred noise spectrum, $S(f)$, is the Fourier transform of $s_{n}{ }^{2}(t)$. The noise spectral density of a $\mathrm{Si}_{3} \mathrm{~N}_{4}$-gate $\mathrm{pH}$-sensitive ISFET operating in the feedback mode with $I_{D}=100 \mu \mathrm{A}$ extracted based on (2) using the fast Fourier transform algorithm is depicted in Fig. 3.

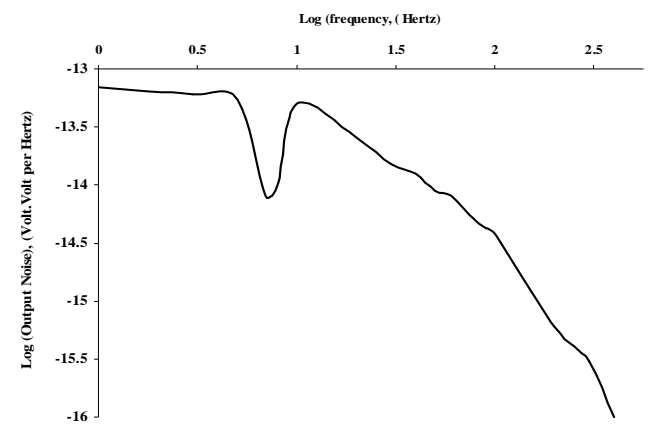

Fig. 3. Extracted ISFET Noise Spectrum

The noise spectrum of Fig. 3 is accurately modeled (coefficient of correlation of 0.993) over the 10-100 Hertz frequency range by an equation of the form, $A / f^{n}$, commonly used to model gate-referred low frequency noise in MOSFET's, with $A=7.31 \times 10^{-13}$ and $n=1.13$. Fig. 3 indicates that roughly below 3 Hertz the output noise spectrum exhibits a diminishing dependence on frequency. Given the fact that the modeled drift data was subtracted from the measured output characteristics of the device, this implies that below a frequency of 3 Hertz, the devices output fluctuations were solely associated with the drift mechanism. Therefore, the appropriate signal segment duration over which the proposed method for sensor signal enhancement may be applied is on the order of a few seconds.

\section{Discussion}

ISFET drift behavior is characterized by an initial relatively fast transient followed by slowly-varying and random-like fluctuations in the device output. The physical model for ISFET drift introduced above, however, reveals the long-term deterministic nature of drift dynamics, and accurately describes drift in terms of growth of a hydration layer given by (1). Differentiation of (1) indicates that over sufficiently small intervals of time, $\Delta t$, on the order of few seconds, the variation in the output due to drift exhibits a weak power-law dependence given by

$$
\Delta s_{d r i f t}(t) \propto \Delta x_{H L}(t) \approx d x_{H L}(t) \propto t^{\beta-1} \exp \left[-(t / \tau)^{\beta}\right]
$$

This is the key feature of the physical model for ISFET drift, which enables sensor signal enhancement by cutting the measuring signal into segments of a few seconds duration over which a relatively fast deterministic response associated with sensor transfer function may be identified.

The ISFET drift behavior following the initial relatively fast transient may be speculated to be associated with low frequency noise. In fact, some authors [14] describe drift as ultra low frequency noise in sensors whose outputs exhibit non-monotonic, slow temporal variations. Conversely, the appearance of $1 / f$ noise at the output of a sensor is very often described as drift [15]. Discrimination between the long-term deterministic drift dynamics and the low frequency $(1 / f)$ noise using the ISFET drift model, however, indicates that the lower limit on the frequency of $\mathrm{pH}$ variations is imposed by drift.

The ISFET drift behavior captured by the proposed physical model may be interpreted as exhibiting hallmarks of chaos. In particular, the observed random-like temporal evolution, characteristic of the long-term deterministic behavior of drift, can be explained in terms of the system's inherent nonlinearity, and is distinct from that associated with fundamental sources of noise such as $1 / f$ noise. This nonlinearity stems from the anomalous dynamics associated with dispersive diffusion according to the proposed model. Specifically, the time dependence of the random walk, which is believed to lead to formation of a hydration layer, is determined by a broad distribution of event times. These event times are identified as hopping times and/or trap release times associated with states in the disordered structure of the $\mathrm{pH}$-sensitive insulator. The distribution of these event times may be regarded as the origin of the long-term drift behavior manifesting as random-like fluctuations in the ISFET drain current following an initial relatively fast transient and persisting, as $t \rightarrow \infty$. These irregular fluctuations, which never repeat exactly, however, have a long-term deterministic character modeled by (1), and indicate that a final thickness for a hydration layer can only be projected after infinitely long times. Finally, as pointed out above, the ISFET drift characteristics are highly sensitive to initial conditions. Nevertheless, experimental drift data do not appear to indicate that in the nonlinear dynamic system described by (1) nearby trajectories separate exponentially fast. That is, the observed drift behavior does not appear to be consistent with a system having a positive Liapunov exponent. Random-looking evolution in time, however, may also occur under conditions that are weaker than that described by a positive Liapunov exponent, in which the separation of nearby trajectories is weaker than exponential [16], [17]. In particular, weakly chaotic systems exhibit anomalous dynamics characterized by novel properties such as ageing, which reflects a weak (power-law) relaxation towards equilibrium [17]. In the case of ISFET drift, which may be interpreted as resulting from ageing of the $\mathrm{pH}$-sensitive insulator surface due to hydration, the anomalous dynamics is associated with the dispersive diffusion leading to a weak power-law relaxation towards equilibrium, as described by (3). 


\section{CONCLUSION}

A physical model for ISFET drift, which quantitatively accounts for the nonlinear dynamical phenomenon of ISFET drift, was briefly introduced. The utility of this model in a physically-informed method for sensor signal enhancement was described. Furthermore, the distinction between the long-term deterministic drift dynamics and the low frequency $(1 / f)$ noise behavior was demonstrated supporting the argument that the physical mechanisms responsible for $1 / f$ noise and drift are entirely different in ISFET's. Drift may be interpreted as a weakly chaotic behavior, exhibiting anomalous dynamics characterized by ageing, which reflects a weak (power-law) relaxation towards equilibrium.

\section{REFERENCES}

[1] A. L. M. Whorter, "1/f Noise and germanium surface properties," in Semiconductor Surface Physics, Philadelphia: University of Pennsylvania Press 1957.

[2] F. N. Hooge, “1/f Noise,” Physica, vol. 83B, pp. 14, 1976.

[3] A. V. D. Ziel, Noise in Solid State Devices and Circuits, New York: Wiley, 1986

[4] K. K. Hung, P. K. Ko, and C. Hu, "A unified model for the flicker noise in metal-oxide field effect transistors," IEEE Trans. on Electron Devices, vol. 37, no. 3, March 1990.

[5] S. Jamasb, S. D. Collins, and R. L. Smith, "A physically-based model for drift in $\mathrm{Al}_{2} \mathrm{O}_{3}$-gate $\mathrm{pH}$ ISFET's," in Proc. of International Conf. on Solid-State Sensors and Actuators (TRANSDUCERS' 97), June 1997, pp. 1379-1382.

[6] S. Jamasb, S. D. Collins, and R. L. Smith, "A physical model for threshold voltage instability in $\mathrm{Si}_{3} \mathrm{~N}_{4}$-gate $\mathrm{H}^{+}$-sensitive FET's ( $\mathrm{pH}$ ISFET's)," IEEE Trans. on Electron Devices, vol. 45, no. 6, pp. 1239-1245, June 1998.
[7] S. Jamasb, S. D. Collins, and R. L. Smith, "A physical model for drift in pH ISFETs,” Sensors and Actuators B: Chemical, vol. 49, pp. 1-2, 1998, pp. 146-155.

[8] S. Jamasb, S. D. Collins, and R. L. Smith, "Determinatio of low-frequency noise Spectrum in ion-selective field effect transistors (ISFET's) based on a physical model for drift," in Proc. of International Conf. on Modeling and Simulation of Microsystems (MSM 2001), March 2001, pp. 514-517.

[9] J. Kakalios, R. A. Street, and W. B. Jackson, "Stretched-exponential relaxation arising from dispersive diffusion of hydrogen in amorphous silicon,” Phys. Rev. Lett. vol. 53, pp. 1037-1040, 1987.

[10] G. Pfister and H. Scher, "Time-dependent electrical transport in amorphous solids: $\mathrm{As}_{2} \mathrm{Se}_{3}$," Phys. Rev. B., vol. 15, no. 4, pp. 2062, 1977.

[11] S. Jamasb, S. D. Collins, and R. L. Smith, "Correction of instability in ion-selective field effect transistors (ISFET’s) for accurate continuous monitoring of $\mathrm{pH}$," in Proc. of International Conf. of the IEEE Engineering in Medicine and Biology (IEEE/EMBS 1997), Oct. 30-Nov.2 1997, pp. 2337-2340.

[12] S. Jamasb, S. D. Collins, and R. L. Smith, "Accurate continuous monitoring using ISFET-based biosensors based on characterization and modeling of drift and low-frequency noise," in Proc. of International Conf. of the IEEE Engineering in Medicine and Biology (IEEE/EMBS 1998), vol. 20, no. 6, pp. 2864-2867, 1998.

[13] S. Jamasb, "An analytical technique for counteracting drifts in ion-selective field effect transistors (ISFET's),” IEEE Sensors Journal, vol. 4, no. 6, December 2004.

[14] J. Fraden, in Handbook of Modern Sensors, $2^{\text {nd }}$ Edition, Springer-Verlag, New York, 1996.

[15] J. K. Atkinson, R. P. Sion, and E. Sizeland, "The characterization of and compensation through sensors array signal processing techniques of drift and low frequency noise in thick-film semiconductor sensors," Sensors and Actuators A, pp. 41-42, pp. 607-611, 1994.

[16] R. Klages, in Microscopic chaos, fractals and transport in nonequilibrium statistical mechanics, World Scientific, Singapore, 2007.

[17] R. Klages, G. Radons, and I. M. Sokolov, in Anomalous Transport, Wiley-CVH, Weinheim, 2008. 